\title{
The Non-Archimedean Polynomials and Merging of Stratified Knowledge Bases
}

\author{
Jianbing $\mathrm{Ma}^{1}$, Weiru Liu ${ }^{1}$, and Anthony Hunter ${ }^{2}$ \\ 1 School of Electronics, Electrical Engineering and Computer Science \\ Queen's University Belfast Belfast BT7 1NN, UK \\ $\{$ jma03,w. liu\}@qub.ac.uk \\ 2 Department of Computer Science University College London \\ Gower Street London WC1E 6BT, UK \\ a.hunterecs.ucl.ac.uk
}

\begin{abstract}
In this paper, a new algebraic representation by the non-Archimedean fields is proposed to model stratified/ranked knowledge bases. The non-Archimedean representation is in the form of the non-Archimedean polynomials. With the nonArchimedean representation, the most widely used ordering strategies are easily induced and compared. Moreover, a framework of prioritized merging operators using the non-Archimedean representation is presented. It is shown that these merging operators satisfy the prioritized merging properties proposed by Delgrande, Dubois and Lang. In addition, several prioritized merging operators in the literature are proved to be special cases of the framework. Furthermore, the egalitarist fusion of incommensurable ranked bases by Benferhat, Lagrue and Rossit is also derived from the non-Archimedean representation.
\end{abstract}

\section{Introduction}

In many applications, there is a need to combine possibly conflicting information from different sources in order to get coherent knowledge. This is the origin of information/data fusion problem. As a very important part of the data fusion problem, in the last two decades, the merging of knowledge bases has attracted significant attention.

Knowledge bases (KBs) can be flat or stratified/ranked. In a flat KB, all the logical formulae are viewed as equally important. In stratified KBs (SKBs), however, formulae are assigned with different levels of importance (priority). A formula at a higher level is viewed as more important than those at a lower level, while in a ranked KB (RKB), each formula is attached to a rank (e.g., an ordinal number). A formula with a higher rank is more preferred than those with lower ranks.

A significant property of stratified/ranked KBs is that higher level/rank items are more important than lower ones. This property is exploited in all the prioritized merging operators in different forms. That is, each of such merging operators involves a step that captures prioritized information as well as a step that merges knowledge. In this paper, we want to investigate whether there is a unified framework to represent the prioritized information prior considering merging and hence use this unified framework to define prioritized merging operators. To achieve this, we introduce the non-Archimedean fields to represent stratified/ranked KBs. We demonstrate that this representation perfectly captures this property and its format is intuitive. In this way, a merging operator only requires a simple definition since most of the work required for merging has already been encoded in the non-Archimedean representation. 
It appears that simple vectors of integers can also be used to represent Stratified $\mathrm{KBs}$, but they have some major drawbacks. First, it is difficult to represent ranked bases with simple vectors, especially in performing the scalings of ranked bases (Def. 15). Second, although simple vectors can be used to obtain orderings between possible worlds, it is hard to present a unified picture on different ordering strategies. In contrast, our non-Archimedean representation solves these problems easily.

The merging of stratified/ranked KBs has been studied in many papers such as, [B+93,Leh95,Bre04,DDL06,QLB06,BLR07]. The extra knowledge implied in a Stratified $\mathrm{KB}$ can be used to induce a total preorder relation on interpretations, and the three widely used ordering strategies are best out, maxsat and leximin [B+93,Bre04]. In [Bre04], the relationship between the three orderings was studied.

In this paper, we first provide the non-Archimedean polynomial (NAP) representation for Stratified KBs which gives us a clear and unified representation of the three preorder relations on interpretations, and therefore, makes the relationship between them immediately provable.

Second, we propose a family of merging operators for Stratified KBs in terms of NAPs. This family of merging operators captures a wide class of prioritized merging operators. It not only captures several existing prioritized merging operators in the literature, such as the linear and leximin operators, but also identifies new merging operators. Our family of prioritized merging operators is the counterpart of the $\mathrm{DA}^{2}$ family of flat merging operators [KLM04].

When merging prioritized $\mathrm{KBs}$, an issue to be considered is whether this set of bases is commensurable. In fact, most of the merging operators for Stratified KBs proposed so far require that the commensurability assumption is in place. For the incommensurable situation, a method called the egalitarist fusion of Ranked KBs was proposed [BLR07]. It is proved that the egalitarist fusion, obtained from a maximum based ordering that is unchanged in all compatible scalings, is equivalent to a Pareto-like operator. In this paper, we show that our non-Archimedean representation of the ranked bases are sufficient to simulate the egalitarist fusion.

In summary, the main contributions of this paper are as follows. First, this paper provides a uniform framework to represent prioritized information at a higher level than embedding it in concrete merging operators. Second, this paper shows that the NAPs provides a unified format to represent three commonly used ordering strategies so that relationship between them can be induced easily. Third, this paper proposes a new family of prioritized merging operators in terms of NAPs which covers a variety of prioritized merging operators in the literature. Fourth, this paper shows that the egalitarist fusion for ranked bases can also be represented and interpreted by NAPs.

The rest of the paper is organized as follows. In Section 2, we recall some basic concepts on propositional logic, Stratified KBs, and non-Archimedean fields. For convenience and subsequent representation, we also introduce some definitions from the $\mathrm{DA}^{2}$ merging operators. In Section 3, we propose the NAPs and relate them to the three ordering strategies. In Section 4, we introduce the framework of prioritized merging operators using NAPs. In Section 5, we give the NAP representation for Ranked KBs and simulate the egalitarist fusion. Finally, we conclude the paper in Section 6. 


\section{Preliminaries}

In this paper, we consider a propositional language $\mathcal{L}_{\mathcal{P S}}$ defined on a finite set $\mathcal{P S}$ of propositional atoms, denoted by $p, q, r$ etc. A proposition $\phi$ is constructed by propositional atoms with logical connectives $\neg, \wedge, \vee, \rightarrow$ in the usual way. An interpretation $w$ (or possible world) is a function that maps $\mathcal{P} \mathcal{S}$ to $\{0,1\}$. The set of all possible interpretations on $\mathcal{P} \mathcal{S}$ is denoted as $W$. Function $w$ can be extended to any propositional sentence in $\mathcal{L}_{\mathcal{P S}}$ in the usual way, $w: \mathcal{L}_{\mathcal{P S}} \rightarrow\{0,1\} . w$ is a model of (or satisfies) $\phi$ iff $w(\phi)=1$, denoted as $w \models \phi$. We use $\operatorname{Mod}(\phi)$ to denote the set of models for $\phi$.

For any set $A$, a pre-order $\leq$ is a reflexive and transitive relation over $A \times A$. $\leq$ is total iff for all elements $a, b \in A$, either $a \leq b$ or $b \leq a$ holds. Conventionally, a strict order $<$ and an indifferent relation $=$ can be induced by $\leq$ such that $\forall a, b \in A, a<b$ iff $a \leq b$ but $b \not \leq a$, and $a=b$ iff $a \leq b$ and $b \leq a$. We use $\max (A, \leq)$ to denote the set $\{a \in A \mid \nexists b \in A, b>a\}$ and $\min (A, \leq)$ for $\{a \in A \mid \nexists b \in A, b<a\}$.

\subsection{Flat/Stratified/Prioritized KBs}

A (flat) $K B K$ is a finite set of propositions. $K$ is consistent iff there is at least one interpretation that satisfies all propositions in $K$.

A $S K B K$ is a set of propositions with a pre-order $\leq$ on $K$, where $\phi \leq \varphi$ means $\phi$ is more important (plausible) than $\varphi$. Commonly, it is written as $K=\left(S_{1}, \ldots, S_{n}\right)$ where each $S_{i}$ (called a stratum) is a set of propositions with all the most important (plausible) elements in $K \backslash \bigcup_{j=1}^{i-1} S_{j}$, i.e., $S_{i}=\min \left(K \backslash \bigcup_{j=1}^{i-1} S_{j}, \leq\right)$, where $\phi<\varphi$ if $\phi \in S_{i}$ and $\varphi \in \phi_{j}$ s.t., $i<j$.

A knowledge profile $E$ is a multi-set of $\mathrm{KBs}$ such that $E=\left\{K_{1}, \ldots, K_{n}\right\}$ where $K_{i}, 1 \leq i \leq n$, is a flat or stratified KB. $K_{E}=K_{1} \sqcup \ldots \sqcup K_{n}$ denotes the set union of $K_{i} \mathrm{~s}$.

In [DDL06], the concept of prioritized observation base $(P O B)$ is introduced. A POB $K$ is in the form $K=\left\langle\sigma_{1}, \ldots, \sigma_{n}\right\rangle$ with $n \geq 1$, where each $\sigma_{i}$ is a set of propositional formulae with reliability level $i$ and formulae with higher reliability levels are more important than those with lower reliability levels (we require that each $\sigma_{i}$ is not empty without losing generality). Obviously, a POB $K=\left\langle\sigma_{1}, \ldots, \sigma_{n}\right\rangle$ induces a SKB $K=\left(S_{1}, \ldots, S_{n}\right)$ such that $S_{i}=\sigma_{n+1-i}$.

Knowledge from a single source (e.g., an expert) can either be represented as a SKB or a POB. However a POB can also be used to represent a collection of knowledge from multiple sources/observations (e.g., a knowledge profile) as discussed in [DDL06]. In this case, a POB is equivalent to a knowledge profile, that is a POB contains all the formulae from a knowledge profile, and each formula is assigned with a reliability value if $\mathrm{KBs}$ in the profile are stratified.

For simplicity and consistency, in the rest of the paper, we use $K=\left\langle S_{1}, \ldots, S_{n}\right\rangle$ to stand for a POB without explicitly considering priority levels, because we do not need the values of these levels in the rest of the paper. Such a $K$ can be taken as consisting of formulae from a knowledge profile of stratified bases where $S_{1}$ contains all the most reliability formulae. We still use $K=\left(S_{1}, \ldots, S_{n}\right)$ to denote a single SKB. We also follow the notations below for a prioritized base [DDL06].

1. $K_{i \rightarrow j}=\left\langle S_{i}, \ldots, S_{j}\right\rangle, 1 \leq i \leq j \leq n$, particularly $K_{1 \rightarrow n}=K, K_{i}=K_{i \rightarrow i}=S_{i}$.

2. $\bigwedge S_{i}=\bigwedge_{\phi \in S_{i}} \phi, \wedge K_{i \rightarrow j}=\bigwedge_{t=i}^{t=j} S_{t}$. 
3. If $K=\left\langle S_{1}, \ldots, S_{n}\right\rangle$ and $K^{\prime}=\left\langle S_{1}^{\prime}, \ldots, S_{p}^{\prime}\right\rangle$, then $\left(K, K^{\prime}\right)$ is the concatenation of $K$ and $K^{\prime}$ such that $\left(K, K^{\prime}\right)=\left\langle S_{1}, \ldots, S_{n}, S_{1}^{\prime}, \ldots, S_{p}^{\prime}\right\rangle$.

4. $\operatorname{Cons}(K)$ is the set of consistent subsets of $K$, that is, the set of all POBs $K^{\prime}=$ $\left\langle S_{1}^{\prime}, \ldots, S_{n}^{\prime}\right\rangle$ such that $\bigwedge K^{\prime}$ is consistent and $S_{i}^{\prime} \subseteq S_{i}, 1 \leq i \leq n$.

\subsection{Non-Archimedean Field}

Now we give a brief introduction to the non-Archimedean fields [Rob73].

Definition 1 ([Ham99]) An ordered field $\langle\mathbb{F},+, \cdot, 0,1,>\rangle$ is a set $\mathbb{F}$ together with:

1. the two algebraic operations $+($ addition $)$ and $\cdot($ multiplication $)$;

2. the two corresponding identity elements 0 and 1 ;

3. the transitive and irreflexive total order $>$ on $\mathbb{F}$ satisfying $1>0$.

Moreover, the set $\mathbb{F}$ must be closed under + and $\cdot$. Addition and multiplication both have to be commutative and associative; the distributive law must hold. And every element $x \in \mathbb{F}$ must have both an additive inverse $-x$ and a multiplicative inverse $1 / x$, except that $x / 0$ is undefined. The order $>$ must be such that $y>z \leftrightarrow y-z>0$. Also, the set $\mathrm{F}+$ of positive elements in $\mathrm{F}$ must be closed under both addition and multiplication.

Both the real line $\mathbb{R}$ and the rationals $\mathbb{Q}$ are obvious examples of ordered fields.

The name "non-Archimedean" stems from the following Archimedes' Axiom. Axiom 1 For any ordered field $\mathbb{F}$, and $0<a<b, a, b \in \mathbb{F}, \exists n \in \mathbb{N}$, s.t., na $>b$.

Thus a non-Archimedean field is a field dissatisfying the Archimedes' Axiom.

The non-Archimedean fields contain real numbers and also infinite numbers and infinitesimals (infinitely small). In this paper, we adopt the smallest non-Archimedean field generated by combining the real line $\mathbb{R}$ and a single infinitesimal $\epsilon$, denoted as $\mathbb{R}(\epsilon)$ [Rob73]. An infinitesimal $\epsilon$ is positive but smaller than any positive real number. If $\epsilon$ is an infinitesimal, then $\epsilon^{i}$ is also an infinitesimal when $i>0$. Moreover, for any positive real numbers $a, b$, we have $a \epsilon^{i+1}<b \epsilon^{i}$ as $\epsilon<b / a$ (as $b / a$ is a positive real number). Note that if $\epsilon$ is an infinitesimal, then $1 / \epsilon$ is an infinite number (larger than any positive real number), and vice versa. As $\mathbb{R}(\epsilon)$ is an ordered field, it is also closed under + and $\cdot$. Moreover, the usual arithmetic properties also apply in $\mathbb{R}(\epsilon)$.

The non-Archimedean field, especially the non-standard probability (i.e., the probability values can involve infinitesimals), has already been introduced in the literature of uncertainty reasoning. For example, in [Spo88], Spohn demonstrated the relationship between his ordinal conditional function and the non-standard probability. In [Pea94], Pearl used the non-standard probability to model non-monotonic reasoning.

Definition $2\left(\leq^{\epsilon}\right)$ Let $x=\sum_{i=1}^{s} a_{i} \epsilon^{b_{i}}$ and $y=\sum_{j=1}^{t} c_{j} \epsilon^{d_{j}}$ be two polynomial representations of infinitesimals, where all $a_{i}, c_{j}$ are positive real numbers, $b_{i}, d_{j}$ are integers, and $b_{1}<\ldots<b_{s}, d_{1}<\ldots<d_{t}$. We write $x \leq^{\epsilon} y$ iff $b_{1} \geq d_{1}$. For convenience, we also write $0<^{\epsilon} x$ as 0 can be seen as $0=\epsilon^{+\infty}$.

The $\leq{ }^{\epsilon}$ relation is not the usual mathematical $\leq$ relation, rather it aims to compare the order of the infinitesimal $\epsilon$, namely, we view $x$ as $O\left(\epsilon^{b_{1}}\right)$ and $y$ as $O\left(\epsilon^{d_{1}}\right)$.

Example 1 Let $x=2 \epsilon^{2}+4 \epsilon^{4}, y=\epsilon^{2}+3 \epsilon^{3}$, then we have $x={ }^{\epsilon} y$. That is, $x$ and $y$ both can be seen as $O\left(\epsilon^{2}\right)$.

We have the following result.

Proposition 1 Let $x, y$ be two polynomial representations of infinitesimals, we have: if $x<^{\epsilon} y$, then $x<y$, if $x \geq y$, then $x \geq^{\epsilon} y$. 


\subsection{The $\mathrm{DA}^{2}$ Merging Operators}

In [KLM04], a family of merging operators, called the $\mathrm{DA}^{2}$ merging operators, was proposed to generalize both model-based and syntax-based merging operators. The $\mathrm{DA}^{2}$ merging operators, consisting of a distance relation between interpretations and two aggregation functions, are defined below.

Definition 3 ([KLM04], distance) A distance relation between interpretations is a total function $d$ from $W \times W$ to $N$ s.t. for every $w_{1}, w_{2} \in W$

1. $d\left(w_{1}, w_{2}\right)=d\left(w_{2}, w_{1}\right), \quad 2 . d\left(w_{1}, w_{2}\right)=0$ iff $w_{1}=w_{2}$.

The distance $d$ between interpretations can be extended to be a distance between an interpretation and a formula as $d(w, \phi)=\min _{w^{\prime} \models \phi} d\left(w, w^{\prime}\right)$.

Definition 4 ([KLM04], aggregation function) An aggregation function is a total function $\oplus$ associating a nonnegative integer to every finite tuple of nonnegative integers and verifying (non-decreasingness), (minimality) and (identity).

non-decreasingness If $x \leq y$, then $\oplus\left(x_{1}, \ldots, x, \ldots, x_{n}\right) \leq \oplus\left(x_{1}, \ldots, y, \ldots, x_{n}\right)$.

minimality $\oplus\left(x_{1}, \ldots, x_{n}\right)=0$ iff $x_{1}=\ldots=x_{n}=0$.

identity For every nonnegative integer $x, \oplus(x)=x$.

Definition 5 ([KLM04], DA ${ }^{2}$ merging operators) Let d be a distance between interpretations and $\oplus$ and $\odot$ be two aggregation functions. For every knowledge profile $E=$ $\left\{K_{1}, \ldots, K_{n}\right\}$ and every integrity constraint $I C$, a DA $A^{2}$ merging operator $\triangle_{I C}^{d, \oplus, \odot}(E)$ is defined in a model-theoretical way by:

$$
\begin{gathered}
\operatorname{Mod}\left(\triangle_{I C}^{d, \oplus, \odot}(E)\right)=\min \left(I C, \leq_{E}^{d, \oplus, \odot}\right) . \\
\leq_{E}^{d, \oplus, \odot} \text { is defined as } w \leq_{E}^{d, \oplus, \odot} w^{\prime} \text { iff } d(w, E) \leq d\left(w^{\prime}, E\right) \text {, where } \\
d(w, E)=\odot\left(d\left(w, K_{1}\right), \ldots, d\left(w, K_{n}\right)\right), \\
\text { and for every } K_{i}=\left\{\phi_{i, 1}, \ldots, \phi_{i, n_{i}}\right\}, d\left(w, K_{i}\right)=\oplus\left(d\left(w, \phi_{i, 1}\right), \ldots, d\left(w, \phi_{i, n_{i}}\right)\right) .
\end{gathered}
$$

An example of distance function is the drastic distance defined as

$$
d_{D}\left(w_{1}, w_{2}\right)=\left\{\begin{array}{l}
0 \text { if } w_{1}=w_{2}, \\
1 \text { otherwise }
\end{array}\right.
$$

For the drastic distance $d_{D}$, we can easily get $d_{D}(w, \phi)=0$ for $w \models \phi$ and $d_{D}(w, \phi)=$ 1 otherwise. Thus it is the characterization function of $\operatorname{Mod}(\phi)$. The commonly used aggregation functions are $\max$ and sum with usual meanings.

\section{NAP Representation of Interpretations}

In this section, we discuss how to obtain non-Archimedean polynomials (NAPs) from SKBs. With the non-Archimedean fields, we associate each stratum in a stratified base with an infinitesimal of degree $i$ (the level of the stratum in the base), so the prioritized information is represented. We then define a NAP for each interpretation $w$ based on the given SKB making use of the representation of prioritized information. This way, the three ordering strategies can be easily simulated using NAPs of interpretations and their relationships can be easily established and proved.

Let $K=\left(S_{1}, \ldots, S_{n}\right)$ be a SKB, the three widely used ordering strategies are best out, maxsat and leximin and they are defined as follows: 
best out ordering [B+93] Let $r_{B O}(w)=\min _{i}\left\{w \not \forall S_{i}\right\}$. Conventionally, $\min _{i} \emptyset=$ $+\infty . w \leq_{b o} w^{\prime}$ iff $r_{B O}(w) \geq r_{B O}\left(w^{\prime}\right)$.

maxsat ordering [Bre04] Let $r_{M O}(w)=\min _{i}\left\{w \models S_{i}\right\} \cdot w \leq_{\text {maxsat }} w^{\prime}$ iff $r_{M O}(w) \leq$ $r_{M O}\left(w^{\prime}\right)$.

leximin ordering [B+93] Let $K_{i}(w)=\left\{\phi \in S_{i} \mid w \models \phi\right\} . w \leq_{\text {leximin }} w^{\prime}$ iff

1. $\left|K_{i}(w)\right|=\left|K_{i}\left(w^{\prime}\right)\right|$ for all $i$, or

2. $\exists i$ such that $\left|K_{i}(w)\right|>\left|K_{i}\left(w^{\prime}\right)\right|$, and $\left|K_{j}(w)\right|=\left|K_{j}\left(w^{\prime}\right)\right|$ for all $j<i$.

Definition 6 (NAP) Let $K=\left(S_{1}, \ldots, S_{n}\right)$ be a SKB, $d$ be a distance and $\oplus$ be an aggregation function, then the NAP of an interpretation $w$ is defined as

$$
N A_{K}^{d, \oplus}(w)=\sum_{i=1}^{n}\left(d\left(w, S_{i}\right) \epsilon^{i}\right)
$$

where for $S_{i}=\left\{\phi_{i 1}, \ldots, \phi_{i n_{i}}\right\}, d\left(w, S_{i}\right)=\oplus\left(d\left(w, \phi_{i 1}\right), \ldots, d\left(w, \phi_{i n_{i}}\right)\right)$.

Eq. 1 defines a family of NAPs of an interpretation $w$, e.g., $N A_{K}^{d_{D}, \max }(w)$ is one specific polynomial where $d=d_{D}$ and $\oplus=\max$. In the following, when there is no confusion, we simplify $N A_{K}^{d, \oplus}(w)$ as $N A_{K}(w)$.

If a SKB has $\{\top\}$ as its first stratum, then we have the following proposition.

Proposition 2 Let $K$ be a $S K B$, then $\forall w \in W, N A_{(\{\top\}, K)}(w)=\epsilon N A_{K}(w)$.

Now, we use NAPs to induce the above ordering strategies.

Definition 7 (best out simulation) For a SKB K, the best out simulation polynomial $b o(w)$ is defined as bo $(w)=N A_{K}^{d_{D}, \max }(w)$.

When $d=d_{D}$ and $\oplus=\max$ in Def. 6, we have $d\left(w, S_{i}\right)=\max _{\phi \in S_{i}}(d(w, \phi))=$ 0 iff $\forall \phi \in S_{i}, d(w, \phi)=0$ (i.e., $\left.w \models S_{i}\right)$. Therefore, $b o(w)$ is actually simplified as

$$
b o(w)= \begin{cases}\epsilon^{t}+\sum_{i=t+1}^{n} d\left(w, S_{i}\right) \epsilon^{i}, & \text { if } r_{B O}(w)=t<\infty ; \\ 0, & \text { if } r_{B O}(w)=\infty .\end{cases}
$$

$b o(w)$ captures the best out strategy by making the most important stratum that $w$ falsifies as the largest $\epsilon$-term.

In Definition 7, we can also let $\oplus=+$, thus we get $b o^{+}(w)=N A_{K}^{d_{D},+}(w)$. Based on the pre-order $\leq^{\epsilon}$ defined in Definition 2, we have

Proposition $3 w \leq_{b o} w^{\prime}$ iff $b o(w) \leq^{\epsilon} b o\left(w^{\prime}\right)$ iff $b o^{+}(w) \leq^{\epsilon} b o^{+}\left(w^{\prime}\right)$.

Definition 8 (maxsat simulation) For a SKB $K$, the maxsat simulation polynomial maxsat $(w)$ is defined as: maxsat $(w)=\sim N A_{K}^{d_{D}, \max }(w)$ where for a polynomial $x=\sum_{i=1}^{n} a_{i} \epsilon^{i}, \sim x=\sum_{i=1}^{n} \sim a_{i} \epsilon^{i}$ s.t. $\sim a_{i}=1$ if $a_{i}=0$ and $\sim a_{i}=0$ otherwise.

For any $w$, if $r_{M O}(w)=t$, we get $N A_{K}^{d_{D}, \max }(w)=\sum_{i=1}^{t-1} \epsilon^{i}+\sum_{i=t+1}^{n} d\left(w, S_{i}\right) \epsilon^{i}$, then we have $\operatorname{maxsat}(w)=\sim N A_{K}^{d_{D}, \max }(w)=\epsilon^{t}+\sum_{i=t+1}^{n} \sim d\left(w, S_{i}\right) \epsilon^{i}$.

maxsat $(w)$ captures the maxsat strategy by making the most important stratum that $w$ satisfies as the largest $\epsilon$-term from the $\sim$ operation.

Proposition $4 w \leq_{\text {maxsat }} w^{\prime}$ iff maxsat $(w) \geq^{\epsilon} \operatorname{maxsat}\left(w^{\prime}\right)$. 
From Definitions 7 and 8 , given any stratified base $K$, the following should hold

$$
b o(w)+\operatorname{maxsat}(w)=\sum_{i=1}^{n} \epsilon^{i} .
$$

For the leximin ordering strategy, we also define the leximin simulation.

Definition 9 (leximin simulation) For a SKB $K$, the leximin simulation polynomial leximin $(w)$ is defined as leximin $(w)=N A_{K}^{d_{D},+}(w)$.

Obviously, we have $b^{+}(w)=\operatorname{leximin}(w)$. As for leximin simulation, because of its lexicographic nature, we cannot use the $\leq^{\epsilon}$ relation to compare two leximin simulation polynomials, instead, the usual mathematical comparative relation $\leq$ should be used. Namely, we have the following result.

Proposition $5 w \leq_{\text {leximin }} w^{\prime}$ iff $\operatorname{leximin}(w) \leq \operatorname{leximin}\left(w^{\prime}\right)$.

With the help of NAPs, the three ordering strategies are represented in a very similar form as shown by Propositions 3, 4 and 5, and the results in the following proposition are immediate following Proposition 1 and Equation 2.

Proposition 6 [Bre04] Let $w, w^{\prime} \in W$, then the following relationships hold:

1. $w<_{b o} w^{\prime}$ implies $w<_{\text {leximin }} w^{\prime}$.

2. $w<_{\text {bo }} w^{\prime}$ implies $w \leq_{\text {maxsat }} w^{\prime}$ and $w<_{\text {maxsat }} w^{\prime}$ implies $w \leq_{b o} w^{\prime}$.

In fact, as $b o^{+}(w)=\operatorname{leximin}(w)$, from $w<_{b o} w^{\prime}$, we get leximin $(w)<^{\epsilon} \operatorname{leximin}\left(w^{\prime}\right)$, thus from Proposition 1, we immediately get $\operatorname{leximin}(w)<\operatorname{leximin}\left(w^{\prime}\right)$ which implies $w<_{\text {leximin }} w^{\prime}$. From $w<_{b o} w^{\prime}$ and Proposition 1, we get $b o(w)<b o\left(w^{\prime}\right)$, from Equation 2, we have $\operatorname{maxsat}(w)>\operatorname{maxsat}\left(w^{\prime}\right)$, thus Proposition 1 gives $\operatorname{maxsat}(w) \geq^{\epsilon}$ $\operatorname{maxsat}\left(w^{\prime}\right)$ which implies $w \leq_{\text {maxsat }} w^{\prime}$.

Example 2 Let $K=\{\{p\},\{q\},\{\neg p \wedge \neg q\},\{\neg p \wedge q\}\}$ be a $S K B$, and let $w=\neg p \wedge q$, $w^{\prime}=\neg p \wedge \neg q$ be two possible worlds. Then we have

$\operatorname{bo}(w)=\operatorname{leximin}(w)=\epsilon+\epsilon^{3}, \operatorname{maxsat}(w)=\epsilon^{2}+\epsilon^{4}$,

bo $\left(w^{\prime}\right)=\operatorname{leximin}\left(w^{\prime}\right)=\epsilon+\epsilon^{2}+\epsilon^{4}, \operatorname{maxsat}\left(w^{\prime}\right)=\epsilon^{3}$.

Hence we get $w={ }_{b o} w^{\prime}, w<_{\max a t} w^{\prime}$ and $w<_{\text {leximin }} w^{\prime}$.

\section{The Non-Archimedean Polynomial Merging Operators}

Since a prioritized observation base (POB) is taken as containing formulae from a set of $\mathrm{SKBs}$, the issue of merging a set of SKBs becomes manipulating formulae in a single POB to obtain a consistent formula (or a set of consistent formulae). To this end, we define the non-Archimedean polynomial (np for short) merging operators for a POB as follows (similar to Def. 5).

Definition 10 Let $d$ be a distance relation between interpretations and $\oplus$ be an aggregation function. For a POB $K=\left\langle S_{1}, \ldots, S_{n}\right\rangle$, a np merging operator $\triangle^{d, \oplus}(K)$ is defined in a model-theoretical way by:

$$
\operatorname{Mod}\left(\triangle^{d, \oplus}(K)\right)=\min \left(W, \leq_{K}^{d, \oplus}\right) .
$$

$\leq_{K}^{d, \oplus}$ is defined as $w \leq_{K}^{d, \oplus} w^{\prime}$ iff $N A_{K}(w) \leq N A_{K}\left(w^{\prime}\right)$, where $N A_{K}$ is the NAP for POB K by Definition 6. 
Since the syntactic form of a POB $K$ is the same as that of a SKB $K^{\prime}$, we can define the NAP $N A_{K}(w)$ from a POB $K$ based on the definition $N A_{K^{\prime}}(w)$ from a SKB $K^{\prime}$.

When $d$ and $\oplus$ are assigned with different distances and different aggregation functions respectively, we get a family of prioritized merging operators.

Other families of prioritized merging operators can be obtainable in terms of NAPs. For example, $\preceq_{K}^{d, \oplus}$ defined as $w \preceq_{K}^{d, \oplus} w^{\prime}$ iff $N A_{K}(w) \leq^{\epsilon} N A_{K}\left(w^{\prime}\right)$ gives us a new family of prioritized merging operators. Due to space limitation, in this paper we only consider the np merging operator defined in Definition 10.

A number of desirable properties for prioritized merging were proposed in [DDL06]. Let $K$ be a POB and $\triangle$ be a prioritized merging operator, these properties are

PMon For every $i<n, \triangle\left(K_{1 \rightarrow i+1}\right) \vdash \triangle\left(K_{1 \rightarrow i}\right)$.

Succ $\triangle(K) \vdash \triangle\left(K_{1}\right)$.

Cons $\triangle(K)$ is consistent.

Taut $\triangle(\{\top\}, K)=\triangle(K)$.

Opt If $\bigwedge K$ is consistent, then $\triangle(K)=\bigwedge(K)$.

IS If $K \equiv K^{\prime}$, then $\triangle(K)=\triangle\left(K^{\prime}\right)$.

RA $\triangle\left(K_{1 \rightarrow i}\right)=\triangle\left(\triangle\left(K_{1 \rightarrow i-1}\right), K_{i}\right)$.

Note: because we represent a prioritized base as $K=\left\langle S_{1}, \ldots, S_{n}\right\rangle$ with $S_{1}$ having the most reliable formulae, and $S_{1}$ is equivalent to $\sigma_{n}$ in the original definition of a prioritized base, the RA (Right Associativity) property looks like a Left Associativity property.

Proposition 7 Let $\triangle^{d, \oplus}$ be any np merging operator defined in Definition 10, then it satisfies (PMon), (Succ), (Cons), (Taut), (Opt), (IS) and (RA).

Some existing prioritized merging operators, e.g., the $\triangle_{\text {linear }}$ and the $\triangle_{\text {leximin }}$ merging operators defined below are special cases of $\triangle^{d, \oplus}$.

Definition 11 (linear, [DP91,Neb94]) Let $K=\left\langle S_{1}, \ldots, S_{n}\right\rangle$, and $\triangle_{\text {linear }}$ be defined inductively by: $\triangle_{\text {linear }}()=\top$, and for $j \geq 1$,

$\triangle_{\text {linear }}\left(K_{1 \rightarrow j}\right)= \begin{cases}\bigwedge S_{j} \bigwedge \triangle_{\text {linear }}\left(K_{1 \rightarrow j-1}\right) & \text { if consistent }, \\ \triangle_{\text {linear }}\left(K_{1 \rightarrow j-1}\right) & \text { otherwise. }\end{cases}$

Definition 12 (leximin, [B+93,Leh95]) Let $K=\left\langle S_{1}, \ldots, S_{n}\right\rangle$. For $K^{1}, K^{2} \in \operatorname{Cons}(K)$, define $K^{2}>_{\text {leximin }} K^{1}$ iff $\exists j$ such that

1. $\left|K_{1 \rightarrow j} \cap K^{2}\right|>\left|K_{1 \rightarrow j} \cap K^{1}\right|$,

2. $\forall i<j,\left|K_{1 \rightarrow i} \cap K^{2}\right|=\left|K_{1 \rightarrow i} \cap K^{1}\right|$.

Then $\triangle_{\text {leximin }}(K)=\bigvee\left\{\bigwedge K^{\prime}\right.$, s.t., $\left.K^{\prime} \in \operatorname{Max}\left(>_{\text {leximin }}, \operatorname{Cons}(K)\right)\right\}$.

Proposition 8 Let $d=d_{D}$ and $\oplus=\max$ in Definition 10 , then $\triangle^{d, \oplus}=\triangle_{\text {linear }}$.

Proposition 9 Let $d=d_{D}$ and $\oplus=+$ in Definition 10, then $\triangle^{d, \oplus}=\triangle_{\text {leximin }}$

However, not all the prioritized merging operators proposed so far in the literature can be induced from the family of $\mathrm{np}$ merging operators. For instance,the discrimin operator [B+93], as it makes use of set inclusion, cannot be represented by NAPs. 


\section{Non-Archimedean Polynomial for Merging RKBs}

In this section, we use NAPs to represent the merging of RKBs. That is, we aim to represent and further interpret the Egalitarist Fusion of incommensurable RKBs [BLR07].

A $R K B K$ is a set of ranked propositions, i.e., $K=\left\{\left(\phi_{1}, r_{1}\right), \ldots,\left(\phi_{n}, r_{n}\right)\right\} . r_{i}$ is the rank of $\phi_{i}, r_{i} \in \mathbb{N} \cup\{+\infty\}, 1 \leq i \leq n$. Here a proposition with a higher rank is more important (prioritized) than the one with a lower rank. The notion of RKB is a generalization of SKBs. Each RKB induces a SKB in which formulae with the same rank are in the same stratum and formulae with the highest rank are in the first stratum. First, we recall some results in [BLR07].

Definition 13 (Ranking functions) A ranking function $\kappa_{K}$ associated with a RKB $K$ is a function: $W \rightarrow \mathbb{N} \cup\{0\}$ such that:

$$
\kappa_{K}(w)= \begin{cases}0 & \text { if } \forall\left(\phi_{i}, r_{i}\right) \in K, w \models \phi_{i}, \\ \max \left(r_{i}: w \not \models \phi_{i}\right) & \text { otherwise. }\end{cases}
$$

With the help of $\kappa_{K}(w)$, a strict order $\triangleleft_{M a x}^{E}$ can be defined between interpretations.

Definition 14 [BLR07] Let $E=\left\{K_{1}, \ldots, K_{n}\right\}$ be a knowledge profile of RKBs and $w, w^{\prime} \in W$ be two interpretations, then we have:

$$
w \triangleleft_{M a x}^{E} w^{\prime} \text { iff } \max \left(\kappa_{K_{i}}(w): 1 \leq i \leq n\right)<\max \left(\kappa_{K_{i}}\left(w^{\prime}\right): 1 \leq i \leq n\right) .
$$

Example 3 Let $E=\left\{K_{1}, K_{2}\right\}$ such that $K_{1}=\{(p, 4),(\neg q, 2)\}$ and $K_{2}=\{(q, 3),(p, 1)\}$,

\begin{tabular}{|c|c|c|c|c|}
\hline & $p q$ & $\kappa_{K_{1}}(w)$ & $\kappa_{K_{2}}(w)$ & $\max$ \\
\hline$w_{0}$ & 100 & 4 & 3 & 4 \\
\hline$w_{1}$ & 01 & 4 & 1 & 4 \\
\hline$w_{2}$ & 10 & 0 & 3 & 3 \\
\hline$w_{3}$ & 11 & 2 & 0 & 2 \\
\hline
\end{tabular}
then we have the following

Obviously, $w_{3}$ is the smallest w.r.t. $\triangleleft_{\text {Max }}^{E}$.

In [BLR07], it is explicitly stated that the scales used in different RKBs are not required to be commensurable. To merge incommensurable ranked bases, a scaling method is proposed as follows.

Definition 15 ([BLR07], Compatible scaling) Let $E=\left\{K_{1}, \ldots, K_{n}\right\}$ be a profile of ranked bases, a scaling $\mathcal{S}$ is defined as $(\bigsqcup$ represents the union of multi-sets):

$$
\mathcal{S}: K_{1} \bigsqcup \ldots \bigsqcup K_{n} \rightarrow \mathbb{N} \quad\left(\phi_{i j}, r_{i j}\right) \mapsto \mathcal{S}\left(\phi_{i j}\right)
$$

$\mathcal{S}$ is said to be compatible with $E$ iff $\forall K_{i} \in E$, we have $\forall(\phi, r),\left(\phi^{\prime}, r^{\prime}\right) \in K_{i}$, $r \leq r^{\prime}$ iff $\mathcal{S}(\phi) \leq \mathcal{S}\left(\phi^{\prime}\right)$.

Given a compatible scaling $\mathcal{S}, K^{\mathcal{S}}$ (resp. $E^{\mathcal{S}}$ ) is used to denote the ranked base (resp. profile of ranked bases) obtained from $K$ (resp. $E$ ) by replacing each pair $\left(\phi_{i}, r_{i}\right)$ with $\left(\phi_{i}, \mathcal{S}\left(\phi_{i}\right)\right)$ (resp. replacing each $K_{i} \in E$ with $K_{i}^{\mathcal{S}}$ ). 
Example 4 (Exam. 3 cont.) Let $E=\left\{K_{1}, K_{2}\right\}$, then a scaling $s_{1}$ produces $K_{1}^{s_{1}}=$ $\{(p, 2),(\neg q, 1)\}$ and $K_{2}^{s_{1}}=\{(q, 5),(p, 2)\}$ is a compatible scaling. However, a scaling $s_{2}$ with $K_{1}^{s_{2}}=\{(p, 2),(\neg q, 3)\}$ and $K_{2}^{s_{2}}=\{(q, 5),(p, 2)\}$ is not a compatible scaling as $s_{2}(p)<s_{2}(\neg q)$ for $K_{1}^{s_{2}}$.

Definition 16 ([BLR07], Compatible scaling ordering) Let $E=\left\{K_{1}, \ldots, K_{n}\right\}, \mathbb{S}_{E}$ be the set of all compatible scalings with $E$, then a partial order $<_{\forall}^{E}$ is defined on $W$ as

$$
\forall w, w^{\prime} \in W, w<_{\forall}^{E} w^{\prime} \text { iff } \forall \mathcal{S} \in \mathbb{S}_{E}, w \triangleleft_{\text {Max }}^{E^{\mathcal{S}}} w^{\prime} .
$$

Example 5 (Exam. 3 cont.) In Example 3, we have $w_{2} \triangleleft_{M a x}^{E} w_{1}$. But after using the compatible scaling $s_{1}$ in Example 4, we get $w_{1} \triangleleft_{M a x}^{E^{s_{1}}} w_{2}$, thus $w_{2} \nless_{\forall}^{E} w_{1}$. It shows the difference between $\triangleleft_{M a x}^{E}$ and $<_{\forall}^{E}$.

Definition 17 ([BLR07], Pareto-like ordering) Let $E=\left\{K_{1}, \ldots, K_{n}\right\}$, we denote $w \triangleleft_{\text {Pareto }} w^{\prime}$ iff the following conditions are satisfied:

1. $\exists i \in\{1, \ldots, n\}, \kappa_{K_{i}}\left(w^{\prime}\right) \neq 0$.

2. $\forall i \in\{1, \ldots, n\}, \kappa_{K_{i}}(w)=\kappa_{K_{i}}\left(w^{\prime}\right)=0$, or $\kappa_{K_{i}}(w)<\kappa_{K_{i}}\left(w^{\prime}\right)$.

The main result in [BLR07] is the proof of equivalence between Pareto-like ordering and the compatible scaling ordering as stated in the following proposition.

Proposition 10 ([BLR07], Prop. 16) $\forall w, w^{\prime} \in W$, we have $w<_{\forall}^{E} w^{\prime}$ iff $w \triangleleft_{\text {Pareto }} w^{\prime}$.

Here we show that strict orders $\triangleleft_{M a x}^{E}$ and $<_{\forall}^{E}$ can be represented by NAPs.

Definition 18 (NAPs from RKBS) Let $d$ be a distance function, for every $R K B K=$ $\left\{\left(\phi_{1}, r_{1}\right), \ldots,\left(\phi_{n}, r_{n}\right)\right\}$, we define the NAP of an interpretation $w$ as $N A_{K}^{d}(w)=$ $\sum_{i=1}^{n}\left(d\left(w, \phi_{i}\right) \epsilon^{-r_{i}}\right)$.

When a SKB $K=\left(S_{1}, \ldots, S_{n}\right)$ is viewed as a RKB

$K^{*}=\left\{\left(\phi_{11},-1\right), \ldots,\left(\phi_{1\left|S_{1}\right|},-1\right), \ldots,\left(\phi_{n 1},-n\right), \ldots,\left(\phi_{n\left|S_{n}\right|},-n\right)\right\}$

where $\phi_{i j} \in S_{i}, 1 \leq j \leq\left|S_{i}\right|, 1 \leq i \leq n$, the NAPs from $K$ is exactly the same as that from $K^{*}$, thus the above definition derives Definition 6 when the aggregation operation $\oplus$ is '+'. For simplicity, we write $N A_{i}(w)$ instead of $N A_{K_{i}}^{d}(w)$, and use $N A_{E}(w)$ for $\sum_{i=1}^{|E|} N A_{i}(w)$ in the rest of the section.

Definition 19 (non-Archimedean pre-order relation) Let $E=\left\{K_{1}, \ldots, K_{n}\right\}$ and $w, w^{\prime}$ be two interpretations. We denote $w<_{N A}^{K_{i}} w^{\prime}$ iff $N A_{i}(w)<N A_{i}\left(w^{\prime}\right) . w<_{N A}^{E} w^{\prime}$ iff $N A_{E}(w)<^{\epsilon} N A_{E}\left(w^{\prime}\right)$.

Note that $<_{N A}^{K_{i}}$ deploys $<$ while $<_{N A}^{E}$ deploys $<^{\epsilon}$.

Example 6 (Exam. 3 Cont.) Let $E=\left\{K_{1}, K_{2}\right\}$, then we have the following

\begin{tabular}{|c|c|c|c|c|}
\hline & $p q$ & $N A_{1}(w)$ & $N A_{2}(w)$ & $N A_{E}(w)$ \\
\hline$w_{0}$ & $\begin{array}{lll}0 & 0\end{array}$ & $\epsilon^{-4}$ & $\epsilon^{-3}+\epsilon^{-1}$ & $\epsilon^{-4}+\epsilon^{-3}+\epsilon^{-1}$ \\
\hline$w_{1}$ & 01 & $\epsilon^{-4}+\epsilon^{-2}$ & $\epsilon^{-1}$ & $\epsilon^{-4}+\epsilon^{-2}+\epsilon^{-1}$ \\
\hline$w_{2}$ & 20 & 0 & $\epsilon^{-3}$ & $\epsilon^{-3}$ \\
\hline$w_{3}$ & $3\left|\begin{array}{ll}1 & 1\end{array}\right|$ & $\epsilon^{-2}$ & 0 & $\epsilon^{-2}$ \\
\hline
\end{tabular}

We can see that $w_{0}<{ }_{N A}^{K_{1}} w_{1}, w_{0}>_{N A}^{K_{2}} w_{1}$, etc., and $w_{3}$ is the smallest w.r.t. $<_{N A}^{E}$. 
Definition 20 Let $E=\left\{K_{1}, \ldots, K_{n}\right\}$ and $w, w^{\prime}$ be two interpretations. We denote $w<<_{N A}^{C o m} w^{\prime}$ iff $\sum_{i=1}^{n} a_{i} N A_{i}(w)<^{\epsilon} \sum_{i=1}^{n} a_{i} N A_{i}\left(w^{\prime}\right)$ where $a_{i}=\epsilon^{\kappa_{K_{i}}\left(w^{\prime}\right)}$ is $a$ commensurable coefficient.

We call $<_{N A}^{C o m}$ the commensurable non-Archimedean pre-order relation because for each $i, \epsilon^{\kappa K_{i}\left(w^{\prime}\right)} N A_{i}\left(w^{\prime}\right)$ has a minimum degree 0 for $\epsilon$. That is, for any $i, j \in$ $[1, n], \epsilon^{\kappa_{K_{i}}\left(w^{\prime}\right)} N A_{i}\left(w^{\prime}\right)$ and $\epsilon^{\kappa_{K_{j}}\left(w^{\prime}\right)} N A_{j}\left(w^{\prime}\right)$ are somehow commensurable. This is illustrated by the following simple example.

Example 7 Let $E=\left\{K_{1}, K_{2}\right\}$ s.t. $K_{1}=\{(p, 4),(\neg q, 2)\}$ and $K_{2}=\{(q, 3),(p, 1)\}$ and $w^{\prime}=\neg p \wedge q$ be a possible world. We have $N A_{1}\left(w^{\prime}\right)=\epsilon^{-2}+\epsilon^{-4}$, and $N A_{2}\left(w^{\prime}\right)=$ $\epsilon^{-1}$. Obviously, the minimum degrees for $\epsilon$ in $N A_{1}\left(w^{\prime}\right)$ and in $N A_{2}\left(w^{\prime}\right)$ are not the same. Now as $\kappa_{K_{1}}\left(w^{\prime}\right)=4$ and $\kappa_{K_{2}}\left(w^{\prime}\right)=1$, we get $\epsilon^{\kappa_{K_{1}}\left(w^{\prime}\right)} N A_{1}\left(w^{\prime}\right)=1+\epsilon^{2}$ and $\epsilon^{\kappa_{K_{2}}\left(w^{\prime}\right)} N A_{2}\left(w^{\prime}\right)=1$, both having 0 as the minimum degree for $\epsilon\left(\right.$ as $\left.1=\epsilon^{0}\right)$.

The following theorems show that the egalitarist fusion can be characterized by the non-Archimedean pre-orders.

Theorem 1 Let E be a profile of $R K B s, \forall w, w^{\prime} \in W$, we have $w \triangleleft_{M a x}^{E} w^{\prime}$ iff $w<{ }_{N A}^{E} w^{\prime}$.

Theorem 2 Let $E$ be a profile of $R K B s, \forall w, w^{\prime} \in W$, we have $w<_{\forall}^{E} w^{\prime}$ iff $w \triangleleft$ Pareto $w^{\prime}$ and iff $w<{ }_{N A}^{\text {Com }} w^{\prime}$.

These theorems show that the egalitarist fusion for the incommensurable RKBs can be described by our non-Archimedean approaches. Furthermore, from Theorem 2, it shows that the egalitarist fusion can in particular be described by our commensurable pre-order relation. Therefore, it is not surprising why the egalitarist fusion does not need the commensurable assumption to deal with the incommensurable RKBs.

\section{Conclusion}

In this paper, we have proposed a new method to model stratified/ranked KBs. Unlike the commonly used logical approaches, our method is largely numerical. We used the non-Archimedean representation for stratified/ranked KBs to represent the ordering strategies, to define new merging operators, and to simulate the egalitarist fusion for incommensurable RKBs. This wide range coverage shows that the non-Archimedean representation is very suitable for modeling stratified/ranked KBs.

In [Pap01], a polynomial representation for each possible world $w$ was proposed which associates $w$ and each epistemic state $\Phi$ (which assigns $w$ an ordinal as its weight) with a polynomial $p_{\Phi}(w)=\sum_{i=0}^{n} p_{i}(w) x^{i}$. Here coefficients $p_{i}(w) \in\{0,1\}, 0 \leq i \leq$ $n$, encodes the binary representation, read in reverse order of the weight assigned to $w$ [B+02], e,g, if the weight of $w$ is 6 , then its binary form is 110 , so $p_{0}(w)=0$ and $p_{1}(w)=p_{2}(w)=1$. The interpretation of such polynomials differs from NAPs in the following aspects. Papini's polynomials consider epistemic states and their coefficients are 0 or 1 , representing the binary form of the weight of $w$ provided by the epistemic states whilst NAPs are for SKBs with each coefficient standing for the aggregation result of distances of formulae in the same level to $w$, and is not limited to $\{0,1\}$. Therefore, Papini's polynomial and NAP are very different.

Our new framework of prioritized merging operators based on NAPs is the counterpart of the $\mathrm{DA}^{2}$ [KLM04] framework of flat merging operators. For each stratum of stratified bases, NAP representation also uses the distance and aggregation function 
to obtain an aggregated effect which is also used in the $\mathrm{DA}^{2}$ framework. Thus, our framework can be seen as an extension of the $\mathrm{DA}^{2}$ framework for SKBs.

The non-Archimedean field is not an entirely new idea in artificial intelligence research. It appeared in the nonstandard probabilities [Spo88,Pea94], in decision making [Leh98] to model utilities to provide a unified theory for qualitative and quantitative decision theories, and in data envelopment analysis [TN09] to define merit functions. However, it has never been used to manipulate stratified/ranked KBs. Our work therefore is novel and significant. There are still many aspects that can be further developed, such as, the relationship between various prioritized merging operators in the literature.

\section{References}

[B+93] S Benferhat, C Cayrol, D Dubois, J Lang, and H Prade. Inconsistency management and prioritized syntax-based entailment. Proc. of IJCAI'93, 640-647, 1993.

[B+02] S Benferhat, D Dubois, S Lagrue, and O Papini. Making revision reversible: an approach based on polynomials. Fundamenta Informaticae, 53(3-4):251-280, 2002.

[BLR07] S Benferhat, S Lagrue, and J Rossit. An Egalitarist Fusion of Incommensurable Ranked Belief Bases under Constraints. Proc. of AAAI'07, 367-372, 2007.

[Bre04] G Brewka. A rank based description language for qualitative preferences. Proc.of ECAI'04, 303-307, 2004.

[DDL06] J Delgrande, D Dubois, and J Lang. Iterated revision as prioritized merging. Proc. of KR'06, 210-220, 2006.

[DP91] D Dubois and H Prade. Epistemic entrenchment and possibilistic logic. Artificial Intelligence, 50:223-239, 1991.

[Ham99] P J Hammond. Non-Archimedean Subjective Probabilities in Decision Theory and Games. Mathematical Social Sciences, 38(2):139-156, 1999.

[KLM04] S Konieczny, J Lang, and P Marquis. DA ${ }^{2}$ merging operators. Artif. Intel., 157:4979, 2004.

[Leh95] D Lehmann. Another perspective on default reasoning. Annals of Mathematics and Art. Int., 15(1):61-82, 1995.

[Leh98] D Lehmann. Nonstandard numbers for qualitative decision making. Proc. of TARK'98, 161-174, 1998.

[Neb94] B Nebel. Base revision operations and schemes: Semantics, representation, and complexity. Proc. of ECAI'94, 341-345, 1994.

[Pap01] O Papini. Iterated Revision Operations Stemming from the History of an Agent's Observations. In H.Rott and M.Williams, editors, Frontiers of Belief Revision, 279301, Kluwer Academic, 2001.

[Pea94] J Pearl. From Adams conditionals to default expressions, causal conditionals and coounterfactuals. In Eells, E., and Skyrms, B., eds., Probability and Conditionals: Belief Revision and Rational Decision, 1994.

[QLB06] G Qi, W Liu, and D A Bell. Merging stratified knowledge bases under constraints. Proc. of AAAI'06, 281-286, 2006.

[Rob73] A Robinson. Function Theory on Some Nonarchimedean Fields. American Mathematical Monthly: Papers in the Foundations of Mathematics, 80:87-109, 1973.

[Spo88] W Spohn. Ordinal Conditional Functions: A Dynamic Theory of Epistemic States. In W.Harper and B.Skyrms (Eds.), Causation in Decision, Belief Change, and Statistics, 2:105-134, Kluwer Academic Publishers, 1988.

[TN09] M Toloo and S Nalchigar. A new integrated DEA model for finding most BCCefficient DMU. Applied Mathematical Modelling, 33(1), 597-604, 2009. 